\title{
Consecuencias de la Pandemia por COVID-19: ¿Pasamos de la obesidad a la desnutrición?
}

\section{Consequences of the COVID-19 Pandemic: Did we pass from obesity to undernutrition?}

\author{
Patricia Gálvez1. https://orcid.org/0000-0001-7147-7058 \\ Daniel Egaña². https://orcid.org/0000-0001-7877-0513 \\ Lorena Rodriguez-Osiac ${ }^{3 *}$. https://orcid.org/0000-0002-7732-2580
}

\begin{abstract}
1. Departamento de Nutrición, Facultad de Medicina, Universidad de Chile, Santiago, Chile, GTOP-UChile. 2. Departamento de Atención Primaria y Salud Familiar, Facultad de Medicina, Universidad de Chile, Santiago, Chile, GTOP-UChile. 3. Escuela de Salud Pública Dr. Salvador Allende G., Universidad de Chile, Santiago, Chile, GTOP-UChile.
\end{abstract}

*Dirigir correspondencia: Lorena Rodríguez Osiac. Escuela de Salud Pública Dr. Salvador Allende G., Universidad de Chile, GTOP-UChile. Independencia 939, Santiago, Chile. Email: Lrodriguezosiac@gmail.com

Recientemente, a través de diversos medios de comunicación masivos se ha relevado el mapa nutricional de la JUNAEB 2020, destacando los preocupantes indicadores de malnutrición infantil'1. Estos datos muestran que la desnutrición ha aumentado en $0,8 \%$ (prevalencia de 2,6\%) respecto a las mediciones de 2019. Por otra parte la malnutrición por exceso (sobrepeso y obesidad) afecta al $54 \%$ de los niños y niñas, $2 \%$ mayor que las mediciones de 2019, destacando el aumento de la obesidad severa de un 6,4\% a un 7,6\% en el mismo periodo'.

Aunque la malnutrición infantil por exceso ha mostrado un crecimiento sostenido en la última década, aumentando casi diez puntos porcentuales ${ }^{2}$, los titulares de medios de comunicación (y algunos expertos) Ilamativamente destacan que debemos preocuparnos porque en Chile está aumentando la desnutrición, condición que parecía erradicada.

¿Tenemos que retroceder en el tiempo y volcar nuestras políticas públicas a erradicar la desnutrición? La respuesta es no. Lo que no significa ignorar ni despreciar los hallazgos, sino considerarlos en su justa medida. Hace una década, la desnutrición infantil registrada por JUNAEB alcanzaba una prevalencia de $2,28 \%{ }^{2}$, sólo un poco menor que la cifra de 2020; y durante estos diez años ha subido y bajado indistintamente. También, es importante considerar que la última versión del mapa nutricional (dado el cierre de las escuelas durante la pandemia), fue construida a partir de mediciones realizadas principalmente en el hogar, por padres/madres u otro cuidador/a del niño o niña. Esta adaptación de la metodología no es menor a la hora de analizar los datos entregados, ya que, dado el tamaño de sus cuerpos, los niñas y niños son muchos más sensibles a centímetros y gramos de más (o de menos) recolectados sin la metodología ni instrumental apropiados. Con esto no queremos negar la situación descrita en el informe de JUNAEB. Pero si es importante que, antes de volcar nuestra preocupación en buscar sol uciones a esta condición de desnutrición infantil, se vuelva a medir al menos a una muestra de niños/as o activar las redes con el sistema de salud, para confirmar los hallazgos y realizar el correcto seguimiento de ellos y ellas.

Por otra parte, nos equivocamos si creemos que no deben preocuparnos las niñas y niños con obesidad, porque durante esta crisis sociosanitaria se está produciendo una dificultad al acceso físico y económico a alimentos sanos. Es muy probable que niñas y niños no estén accediendo a dietas saludables y estén consumiendo una 
alta cantidad de alimentos ultraprocesados y farináceos de bajo costo que promueven la obesidad.

Un porcentaje de nuestros niños y niñas están sufriendo de inseguridad alimentaria, lo cual traería como consecuencia la malnutrición, ya sea por déficit o por exceso. Esto quiere decir, que una parte de los niños y niñas de nuestro país, no están teniendo acceso físico, económico y de manera estable a alimentos sanos, suficientes y seguros ${ }^{3}$. De acuerdo a datos de la encuesta CASEN, previo a la pandemia, un 13,7\% de los hogares del país sufría ya de inseguridad alimentaria ${ }^{4}$, la que, producto de la pandemia durante el primer semestre del año 2020, alcanzo el 19,4\% ${ }^{5}$. Esto como consecuencia del contexto sanitario, donde muchas familias han visto mermados sus ingresos, aumentando la pobreza ${ }^{6}$, resurgiendo las ollas comunes ${ }^{7}$, aumentado de precio algunos alimentos ${ }^{8}$, y cerrándose almacenes y ferias. Al mismo tiempo, con las escuelas suspendidas el programa de alimentación escolar (PAE) se ha reorganizado en la entrega de cajas de alimentos a las familias de las niñas y niños, siendo la mayoría alimentos no perecibles, con muy limitada entrega de frutas y verduras ${ }^{9}$. Aunque el cálculo de la mercadería se hace por niña o niño, esta inevitablemente se consume colectivamente en la familia. No podría ser de otra manera. La consecuencia de todo esto es la falta de nutrientes y no solamente de calorías, que muy probablemente se traduce en cifras más altas de sobrepeso y obesidad, con un déficit oculto de proteínas, vitaminas, minerales y fibra.

Las medidas del gobierno han sido insuficientes para una parte importante de la población, pero la solución no pasa por ponernos a hablar de desnutrición y volcar nuestras políticas públicas a esta condición, sino por tomar medidas efectivas que le permitan a todas las familias, disponer y acceder en todo momento y en todo lugar a alimentos saludables, frescos, naturales y culturalmente pertinentes, por ejemplo a través de subsidiar la compra de verduras, frutas, legumbres y pescados en ferias libres, promover los circuitos cortos de comercialización y proveer a las familias de un ingreso seguro y suficiente que les permita asegurar la adecuada alimentación de todos sus integrantes.

Tabla. Variación Estado Nutricional Escolares JUNAEB 2009-2020.

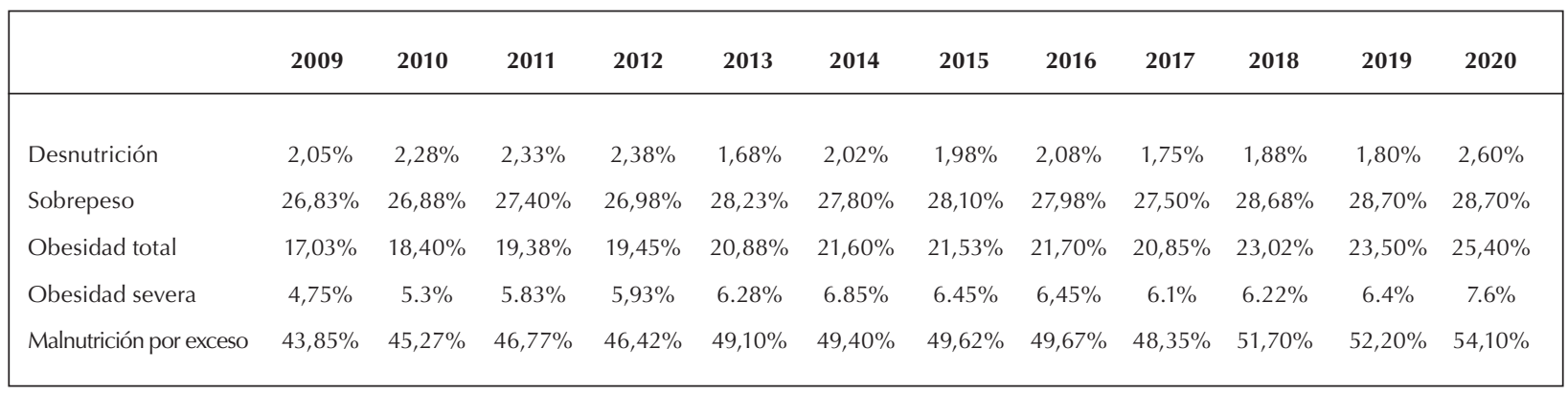

Fuente: Elaboración propia en base a datos de Mapa Nutricional JUNAEB.

\section{REFERENCIAS}

1. UUNAEB. Mapa Nutricional. 2020. https://www.junaeb.cl/wp-content/uploads/2021/03/MapaNutricional2020_.pdf

2. JUNAEB. Mapa Nutricional 2018. Situación nutricional de los párvulos y escolares de establecimientos escolares con financiamiento público del país. 2019. https://www.junaeb.cl/wp-content/uploads/2019/12/Informe-Mapa-Nutricional-2018.pdf

3. FAO. Hambre e inseguridad alimentaria. 2020. http://www.fao.org/hunger/es/

4. Ministerio de Desarrollo Social y Familia. Gobierno de Chile. Inseguridad Alimentaria. Sintesis de resultados. 2017. http://observatorio. ministeriodesarrollosocial.gob.cl/casen-multidimensional/casen/docs/CASEN_2017_Inseguridad_alimentaria.pdf

5. Ministerio de Desarrollo Social. Gobierno de Chile. Encuesta Social COVID-19. 2020. http://observatorio.ministeriodesarrollosocial. gob.cl/storage/docs/covid19/Inseguridad_Alimentaria_Encuesta_Social_Covid-19.pdf

6. CEPAL. Pandemia provoca aumento en los niveles de pobreza sin precedentes en las últimas décadas e impacta fuertemente en la desigualdad y el empleo. 2021. https://www.cepal.org/es/comunicados/pandemia-provoca-aumento-niveles-pobreza-sin-precedentesultimas-decadas-impacta

7. La Olla de Chile, Universidad Alberto Hurtado. Las ollas comunes en pandemia. Estrategias locales ante la crisis del hambre. 2021. http://geografia.uahurtado.cl/las-ollas-comunes-en-pandemia-estrategias-locales-ante-la-crisis-del-hambre/

8. The World Bank. Food Security and COVID-19. 2021. https://www.worldbank.org/en/topic/agriculture/brief/food-security-and-covid-19

9. JUNAEB. Junaeb comienza entrega de alimentación con modalidad mixta para 1.800.000 estudiantes de todo Chile. 2021. https:// www.junaeb.cl/archivos/61989. 